DOI: 10.20472/IAC.2018.935.042

RAED SAHAWNEH

Irbid National University, Jordan

\title{
METHODS AND ALGORITHMS OF SPEECH SIGNALS PROCESSING AND COMPRESSION AND THEIR IMPLEMENTATION IN COMPUTER SYSTEMS
}

\begin{abstract}
:
The review and comparative analysis of the methods of compression and recognition of speech signals is carried out. The result of the carried out analysis of the existing recognition methods indicates, that all of them are based on the use of "inflexible" algorithms, which are badly adapted to the characteristic features of speech signals, thus degrading the efficiency of the operation of the whole recognition system. The necessity of the use of algorithms for determination of recognition features along with the use of the wavelet packet analysis as one of the advanced directions of the creation of the effective methods and principles of the development of the speech signals recognition systems is substantiated.

Analysis of the compression methods with the use of the orthogonal transformations at the complete exception of minimal decomposition factors is conducted; a maximal possible compression degree is defined. In this compression method the orthogonal transformation of the signal segment with the subsequent exception of the set of the smallest modulo decomposition factors, irrespective of the order of their distribution, is conducted. Therefore the additional transfer of the information on the factors distribution is required.

As a result, two information streams appear, the first one corresponds to the information stream on the decomposition factors, and the second stream transfers information on the distribution of these factors.

Method of the determination of the speech signals recognition features and the algorithm for nonlinear time normalization is proposed and proved.

Wavelet-packet transformation is adaptive, i.e. it allows adapting to the signal features more accurately by means of the choice of the proper tree of the optimal decomposition form, which provides the minimal number of wavelet factors at the prescribed accuracy of signal reconstruction, thus eliminating the information-surplus and unnecessary details of the signals.
\end{abstract}

\section{Keywords:}

recognition of speech signals, compression, recognition features.

JEL Classification: C80 